\title{
Chlamydia trachomatis among sexually active teenage girls: influence of sampling location and clinical signs on the detection rate
}

\author{
V A Rahm, V Odlind, H Gnarpe
}

\begin{abstract}
A total of 707 cultures for Chlamydia trachomatis were obtained from healthy teenage girls attending an adolescence clinic for family planning. Apart from sampling from the endocervix, specimens were also obtained from outside the squamocolumnar junction and from the posterior fornix and cultured separately in order to study whether it was possible to improve the detection rate of $C$ trachomatis by sampling from outside the endocervix. In $91(12 \cdot 8 \%)$ of the girls a positive culture was found. Of the chlamydia positive samples, $83.5 \%$ were obtained from the endocervix and $95 \%$ from outside the squamocolumnar junction of the portio. Of the 91 positive cultures, $15(16.5 \%)$ were positive only in the sample obtained from the portio surface. The results indicate that the detection rate of $C$ trachomatis could be improved by extended specimen collection. $C$ trachomatis was isolated significantly more often when there was an ectopy, irrespective of whether the specimens had been collected from the endocervix or from outside the squamocolumnar junction. $C$ trachomatis was particularly common if there was an ectopy and a bleeding at specimen collection. If there was no bleeding at sampling, C trachomatis was equally common in women with as in those without an ectopy
\end{abstract}

$C$ trachomatis has previously been reported to be highly prevalent among young, sexually active girls. ${ }^{1}$ Genital infection with $C$ trachomatis in young women is often asymptomatic and it has not been possible to correlate the clinical findings to infection with any degree of accuracy. ${ }^{12}$

The diagnosis of genital infection with $C$ trachomatis is hampered by limited sensitivity and

Gävle Ungdomsmottagning, Department of Obstetrics and Gynaecology, University of Uppsala, 75185 Uppsala, Sweden

V A Rahm, V Odlind, H Gnarpe accuracy of various diagnostic methods. In order to improve the detection rate of $C$ trachomatis by culture, we have previously studied the influence of the sampling technique and reported that careful cleansing of the cervix before sampling considerably increased the pick-up rate. ${ }^{1}$ Preliminary studies in our clinic have suggested that the detection rate by culture could be further increased by specimen collection from outside the endocervix.

Cervical infection with $C$ trachomatis has been reported to occur more often in women with a visible ectocervical zone of columnar epithelium (ectopy) than in those without such an ectopy..$^{3-6}$

The aim of the present investigation was to find if $C$ trachomatis could be isolated from outside the squamocolumnar junction of the portio and from the posterior fornix and if the detection rate was interrelated with the presence of a cervical ectopy and/or friability (bleeding) at sampling in young sexually active girls.

This report is a part of a larger study, the aim of which is to investigate the prevalence and incidence of $C$ trachomatis among young, sexually active girls, to optimise the detection rate by adjusting sampling technique and to determine various factors that correlate with chlamydial infection in this particular age group.

\section{Material and methods}

Patient sample. A total of 707 samples for chlamydial cultures was obtained from sexually active teenage girls, attending an adolescence clinic for family planning from March 1986 to June 1987. The girls came to the clinic mainly for family planning. Girls who had taken antibiotics within two months were not enrolled in the study. The mean age of the girls was 17.7 years and the age ranges were between 14 and 19 years. Combined oral contraceptive pills were used by $82 \%$ of the girls.

Clinic characteristics. The clinic is a primary health facility which is open to all teenagers in the community and receives approximately 4000 visits per year. The vast majority of visitors are girls who come for birth control counselling. The clinic is served by two midwives with training and experience in birth control and teenage counselling. The girls were 
allocated by chance to one of the two investigators. Clinical examination. A speculum examination was performed and the cervix was visualised and described. Colposcopy was not performed. The extent of the ectopy was scored as no ectopy, moderate (involving less than one fourth of the visible ectocervix), spread (involving one fourth or more of the visible ectocervix). Friability (bleeding in the process of manipulation of the cervix and portio for specimen collection) was noted as none, slight or abundant.

Three specimens for chlamydial culture were obtained, using an ENT swab after careful cleansing of the portio with a large dry cotton swab, according to the following:

(1) From the portio surface. (If there was an ectopy the specimen was obtained from outside the squamocolumnar junction),

(2) From the endocervical canal,

(3) From the posterior fornix.

The swabs were immersed in separate tubes containing 2 SP medium and kept at $+4^{\circ} \mathrm{C}$ until they were transported to the laboratory within 24 hours of sampling or less. Cultures for $C$ trachomatis were made in cycloheximide treated McCoy cells. The results were given as positive or not, and, moreover, the number of infected cells in each separate culture was recorded.

\section{Statistical analysis}

The data were recorded on precoded proformas and analysed with a computer using the Statistical Analysis System (SAS). Tests of significance were performed using the $\chi^{2}$ method with Yates's correction. The McNemar test was also used to compare the difference between the culture results from cervix and portio.

\section{Results}

Of the 707 examinations performed during the period, 91 cultures were chlamydia positive from at least one location (table 1 ).

$C$ trachomatis could be isolated more often from specimens obtained from the portio surface ( 86 cases; $94.5 \%$ ) than from the endocervix (76 cases; $83.5 \%$ ). Portio was the only location from which $C$ trachomatis was isolated in 15 cases and the endocervix was the only location in five cases (table

Table 1. Results of culture in 91 chlamydia infected girls in relation to sampling location

\begin{tabular}{lrrl}
\hline & \multicolumn{3}{c}{ Chlamydia positive } \\
\cline { 2 - 4 } & $n$ & $\%$ & $\begin{array}{l}95 \% \text { confidence } \\
\text { limits }\end{array}$ \\
\hline Cervix/portio & 71 & $78 \cdot 0$ & $\begin{array}{l}69 \cdot 5-86.5 \\
\text { Cervix only }\end{array}$ \\
Portio only & 5 & $5 \cdot 5$ & $0 \cdot 8-10 \cdot 2$ \\
& 15 & 16.5 & $8 \cdot 9-24 \cdot 1$ \\
\hline
\end{tabular}

Table 2 The number and proportion of infected cells as determined per high power field $(\times 120)$ from cultured specimens from the cervix, portio and fornix, respectively

\begin{tabular}{|c|c|c|c|}
\hline Cells & $\begin{array}{l}\text { Cervix } \\
n \%\end{array}$ & $\begin{array}{l}\text { Portio } \\
n \%\end{array}$ & $\begin{array}{l}\text { Fornix } \\
n \%\end{array}$ \\
\hline $\begin{array}{l}1-4 \\
5-9 \\
>10\end{array}$ & $\begin{array}{ll}36 & 47 \cdot 4 \\
15 & 19 \cdot 7 \\
25 & 32 \cdot 9\end{array}$ & $\begin{array}{ll}39 & 45 \cdot 3 \\
17 & 19 \cdot 8 \\
30 & 34 \cdot 9\end{array}$ & $\begin{array}{rr}36 & 72 \cdot 0 \\
11 & 22 \cdot 0 \\
3 & 6 \cdot 0\end{array}$ \\
\hline
\end{tabular}

1). This difference was significant $\left(\chi^{2}=5 \cdot 0\right.$; $\mathrm{p}<0.05)$. A positive culture from the posterior fornix was observed in $50(54.9 \%)$ of the specimens, always in combination with a positive culture from the endocervix and/or portio.

Of the 15 girls who were chlamydia positive only in specimens obtained from the portio surface, six had no visible ectopy, six a moderate and three a spread ectopy. Of the five girls who were chlamydia positive only in endocervix, four had no visible ectopy and one a moderate ectopy.

In order to assess the degree of infection, the number of infected cells was counted in each culture from each location. There was no statistically significant difference between cervix and portio in the number of infected cells. Chlamydia positive cultures from the fornix, however, contained fewer infected cells significantly more often than cultures from the cervix and portio $\left(\chi^{2}=15.02\right.$; df $=2$; p < 0.001; table 2).

$C$ trachomatis was isolated more often in girls with an ectopy. At 308 examinations, where no ectopy was found, $C$ trachomatis was isolated in 28 cases $(9 \cdot 1 \%)$. If an ectopy was present, $C$ trachomatis was isolated from 63 of the remaining 399 patients $(15 \cdot 8 \%$ ). Thus, there was a strong correlation between chlamydial infection and ectopy $\left(\chi^{2}=6.955 ; \mathrm{df}=1\right.$; $\mathrm{p}<0.01$; table 3).

There was no bleeding in connection with specimen collection in $\mathbf{5 0 0}$ of the 707 examinations. A positive chlamydial culture was found in $47(9.4 \%)$ of those. A slight bleeding occurred in 165 examinations and an abundant bleeding in the remaining 42 . In those groups positive chlamydial cultures were

Table 3 The number and rate of chlamydial culture positive samples obtained from cervix only, from both cervix and portio and from portio only, in relation to the presence or absence of an ectopy

\begin{tabular}{|c|c|c|c|c|c|c|c|}
\hline & \multicolumn{7}{|c|}{ Chlamydia positive } \\
\hline & $\begin{array}{l}\text { Cervix } \\
\text { only } \\
n \quad \%\end{array}$ & $\begin{array}{l}C e \\
+I \\
n\end{array}$ & $\begin{array}{l}\text { vix } \\
\text { ortio } \\
\%\end{array}$ & & $\begin{array}{r}\text { rtio } \\
\%\end{array}$ & ${ }_{n}^{T o t}$ & $\%$ \\
\hline $\begin{array}{l}\text { No ectopy } \\
\mathrm{N}=308\end{array}$ & $4 \quad 1 \cdot 3$ & 18 & $5 \cdot 8$ & 6 & 1.9 & 28 & $9 \cdot 1$ \\
\hline $\begin{array}{l}\text { Ectopy } \\
N=399\end{array}$ & $\begin{array}{ll}1 & 0 \cdot 2\end{array}$ & 53 & $13 \cdot 3$ & 9 & $2 \cdot 2$ & 63 & $15 \cdot 7$ \\
\hline
\end{tabular}


Table 4 Bleeding and ectopy in relation to 91 chlamydia positive cultures in 707 examinations

\begin{tabular}{lccc}
\hline & & \multicolumn{2}{c}{ Chlamydia positive } \\
\cline { 3 - 4 } & $n$ & $n$ & $\%$ \\
\hline Bleeding/ectopy & 170 & 40 & 23.5 \\
Bleeding/no ectopy & 37 & 4 & 10.8 \\
No bleeding/ectopy & 229 & 23 & 10.0 \\
No bleeding/no ectopy & 271 & 24 & 8.9 \\
\hline
\end{tabular}

obtained from $30(18.2 \%)$ and $14(33.3 \%)$, respectively. The difference between each of the three groups was significant $\left(\chi^{2}=25 \cdot 2 ; d f=2\right.$; $\mathrm{p}<0.001$ ).

$C$ trachomatis was isolated more often from girls with the combined symptoms of bleeding and ectopy than from those with one or none of those symptoms (table 4). Pairwise comparisons of the groups presented in table 4, using Chi-square statistics, showed statistical difference only between the groups bleeding/ectopy and no bleeding/ectopy, where $C$ trachomatis was found significantly more often in the group with bleeding/ectopy $\left(\chi^{2}=13.34\right.$; $\mathrm{p}<0.001)$. When various log-linear models were fitted to the individual frequencies of ectopy, bleeding and $C$ trachomatis, the strongest correlation was found between bleeding and ectopy $(p<0.001)$ and between bleeding and chlamydial infection $(p<0.001)$ but there was no statistical correlation between ectopy and chlamydial infection. Thus, if there was no bleeding at sampling, chlamydia was equally common in women with as in those without an ectopy.

An analysis of the relation between the degree of bleeding at specimen collection (none, slight, abundant) and the size of the ectopy (none, moderate, spread) and the isolation rate of $C$ trachomatis, suggests that an infection with $C$ trachomatis is particularly common in those with a spread ectopy and an abundant bleeding (table 5).

\section{Discussion}

This study confirms previous reports that $C$ trachomatis is frequently found among asymptomatic young, sexually active women and underlines the need for screening for chlamydial infection in this

Table 5 The total number of girls with/without ectopy and bleeding at sampling and the proportion of chlamydia positive specimens $(C T+\%)$ in relation to the occurrence of ectopy and/or bleeding at sampling

\begin{tabular}{lcccccc}
\hline & \multicolumn{1}{l}{ Ectopy } \\
\cline { 2 - 7 } Bleeding & None & $\begin{array}{l}C T+ \\
\%\end{array}$ & Moderate & $\begin{array}{l}C T+ \\
\%\end{array}$ & Spread & $\begin{array}{l}C T+ \\
\%\end{array}$ \\
\hline None & 271 & 18.9 & 177 & 19.6 & 52 & 11.5 \\
Slight & 37 & 10.8 & 90 & 17.8 & 38 & 26.3 \\
Abundant & 0 & 0 & 14 & 14.3 & 28 & 42.9 \\
\hline
\end{tabular}

group of women. However, for such screening programmes to be cost-effective, it is important both to identify those most at risk within the group and to optimise the diagnostic procedures. This part of the study deals mainly with the latter, showing that a simple adjustment of the sampling technique by obtaining specimens both from the endocervix and portio surface, can significantly increase the number of chlamydia positive cultures. Statistically, if specimens from the portio surface had not been taken, the infection would not have been detected in between 9 and $24 \%$ of the patients $(95 \%$ confidence interval). Similarly, had specimen collection from the endocervix been omitted, between $1 \%$ and $10 \%$ of the patients would not have been diagnosed.

$C$ trachomatis is described as an obligately intracellular bacteria and in man reported to inhabit primarily columnar and pseudostratified columnar epithelium. ${ }^{7}$ This is not in line with our finding that $C$ trachomatis could be isolated in $95 \%$ of all positive cases in samples collected from outside the squamocolumnar junction. Although our results suggest that $C$ trachomatis is as likely to be found in squamous epithelial cells as in columnar cells, their interpretation needs further investigation. Colposcopy was not performed to confirm that sampling was always done from an intact squamous epithelium. Neither can it be ruled out that the culture positive cells obtained from outside the squamocolumnar junction represent discharged cells from adjacent infected epithelium.

An association between chlamydial infection and ectopy has been demonstrated in several studies. ${ }^{3-68}$ Our study confirms the results of others, but, when women with bleeding at sampling were excluded, chlamydial infection was equally common in women without as in those with an ectopy. It is not possible from the present study to ascertain whether a friable ectopy is caused by chlamydial infection or just increases the shedding of $C$ trachomatis from the cervix, thereby making detection by culture easier. Young women are more likely to have an ectopy than older women. ${ }^{9}$ Chlamydial infection is found significantly more often in young women than in women over age 25 years. ${ }^{8}$ It seems justified to speculate that the presence of an ectopy may increase the risk of becoming infected with $C$ trachomatis on exposure and that friability is a consequence of the infection.

In conclusion this study has shown that the location for specimen collection can affect the pickup rate of $C$ trachomatis. Sampling from the portio, irrespective of the presence or absence of a visible ectopy, should be performed to optimise the results of chlamydial culture. The study has also demonstrated that the combined symptoms ectopy and friability of the cervix make a chlamydial infection very likely in otherwise healthy, asymptomatic young women. 
The authors acknowledge the excellent co-operation of the staff at Gävle Ungdomsmottagning, especially that of Mrs Gerd Rosén, nurse-midwife. Statistical assistance was provided by associate professor Roland Pettersson, Department of Statistics, University of Uppsala, which is gratefully acknowledged.

Address for reprints: V A Rahm, Department of Obstetrics and Gynaecology, University of Uppsala, 75185 Uppsala, Sweden

1 Rahm V-A, Gnarpe H, Odlind V. Chlamydia trachomatis among sexually active teenage girls: Lack of correlation between chlamydial infection, history of the patient and clinical signs of infection. Br J Obstet Gynaecol 1988;95:916-9.

2 Thejls H, Rahm VA, Rosén G, Gnarpe H. (1987) Correlation between chlamydia infection and clinical evaluation, vaginal wet smear, and cervical swab test in female adolescents. Am J Obstet Gynecol 1987;157:974-6.
3 Arya OP, Mallinson H, Goddard AD. Epidemiological and clinical correlates of chlamydial infection of the cervix. $\mathrm{Br} J$ Venereal Dis 1981;57:118-24.

4 Chacko MR, Lovchik J. Chlamydia trachomatis infection in sexually active adolescents: Prevalence and risk factors. $J$ Pediatr 1984;73:836-40.

5 Harrison RH, Costin M, Meder J, et al. Cervical Chlamydia trachomatis infection in university women: Relationship to history, contraception, ectopy, and cervicitis. Am J Obstet Gynceol 1985;153:244-51.

6 Louv WC, Austin H, Perlman J, Alexander WJ. Oral contraceptive use and the risk of chlamydial and gonococcal infections. Am J Obstet Gynecol 1989;160:396-402.

7 Thompson SE, Washington EA. Epidemiology of sexually transmitted Chlamydia trachomatis infections. Epidemiol Rev 1983;5:96-123.

8 Handsfield HH, Jasman LL, Roberts PL, Hanson VW, Kothenbeutel RL, Stamm WE. Criteria for selective screening for Chlamydia trachomatis infection in women attending family planning clinics. JAMA 1986;255:1730-4.

9 Singer A. The uterine cervix from adoloscence to the menopause. Br J Obstet Gynaecol 1975;82:81-99.

Accepted for publication 14 November 1989 